\title{
Surgically Placed Abdominal Wall Catheters on Postoperative Analgesia and Outcomes After Living Liver Donation
}

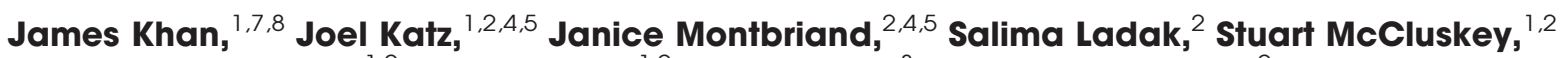 \\ Coimbatore Srinivas, ${ }^{1,2}$ Raynauld Ko, ${ }^{1,2}$ David Grant, ${ }^{3}$ Ashleene Bradbury, ${ }^{2}$ \\ Yannick LeManach, ${ }^{,, 7,8}$ and Hance Clarke ${ }^{1,2}$ \\ ${ }^{1}$ Department of Anesthesia, University of Toronto, Toronto, Canada; ${ }^{2}$ Department of Anesthesia and Pain \\ Management, Toronto General Hospital, and ${ }^{3}$ Multiorgan Transplant Program, University Health Network, \\ Toronto, Canada; ${ }^{4}$ Department of Psychology and ${ }^{5}$ School of Kinesiology and Health Science, York \\ University, Toronto, Canada; Departments of ${ }^{6}$ Anesthesia and ${ }^{7}$ Clinical Epidemiology and Biostatistics, \\ McMaster University, Hamilton, Canada; and ${ }^{8}$ Population Health Research Institute, Hamilton, Canada
}

Living donor liver resections are associated with significant postoperative pain. Epidural analgesia is the gold standard for postoperative pain management, although it is often refused or contraindicated. Surgically placed abdominal wall catheters (AWCs) are a novel pain modality that can potentially provide pain relief for those patients who are unable to receive an epidural. A retrospective review was performed at a single center. Patients were categorized according to their postoperative pain modality: intravenous (IV) patient-controlled analgesia (PCA), AWCs with IV PCA, or patient-controlled epidural analgesia (PCEA). Pain scores, opioid consumption, and outcomes were compared for the first 3 postoperative days. Propensity score matches (PSMs) were performed to adjust for covariates and to confirm the primary analysis. The AWC group had significantly lower mean morphine-equivalent consumption on postoperative day 3 [18.1 mg, standard error (SE) = 3.1 versus 28.2 $\mathrm{mg}, \mathrm{SE}=3.0 ; P=0.02$ ] and mean cumulative morphine-equivalent consumption $(97.2 \mathrm{mg}, \mathrm{SE}=7.2$ versus $121.0 \mathrm{mg}$, SE $=9.1 ; P=0.04)$ in comparison with the IV PCA group; the difference in cumulative-morphine equivalent remained significant in the PSMs. AWC pain scores were higher than those in the PCEA group and were similar to the those in the IV PCA group. The AWC group had a lower incidence of pruritus and a shorter hospital stay in comparison with the PCEA group and had a lower incidence of sedation in comparison with both groups. Time to ambulation, nausea, and vomiting were comparable among all 3 groups. The PSMs confirmed all results except for a decrease in the length of stay in comparison with PCEA. AWCs may be an alternative to epidural analgesia after living donor liver resections. Randomized trials are needed to verify the benefits of AWCs, including the safety and adverse effects.

Received August 29, 2014; accepted December 4, 2014.

Abbreviations: ANOVA, analysis of variance; APS, acute pain service; AUPC, area under the pain curve; AWC, abdominal wall catheter; BMI, body mass index; IV, intravenous; LLDT, living liver donor transplantation; PCA, patient-controlled analgesia; PCEA, patient-controlled epidural analgesia; POD, postoperative day; PONV, postoperative nausea and vomiting; PSM, propensity score match; SE, standard error; TAP, transversus abdominis plane.

James Khan is supported by a masters award from the Canadian Institute of Health Research and a fellowship grant from the Michael G. DeGroote Institute of Pain Research and Care. Hance Clarke is supported by a merit award from the Department of Anesthesia at the University of Toronto and by the Strategic Training for Advanced Genetic Epidemiology Program in Genetic Epidemiology at the Canadian Institute of Health Research.

Potential conflict of interest: Nothing to report.

Address reprint requests to James Khan, B.H.Sc., M.D., Pain Research Unit, Department of Anesthesia and Pain Management, Toronto General Hospital, 200 Elizabeth Street, 3EB 317, Toronto, Ontario M5G 2C4, Canada. Telephone: 416-946-0608; FAX: 416-340-3698;

E-mail: james.khan@medportal.ca 
Liver transplantation is the only option for survival for patients dying from end-stage liver disease. ${ }^{1}$ Unfortunately, eligible transplant organs from deceased donors are scarce, and approximately 5\% to $10 \%$ of patients waiting for a liver transplant will die without receiving a donation. ${ }^{2}$ Living liver donor transplantation (LLDT) programs have become a critical resource for those waiting for a lifesaving transplant.

Living donors are relatively healthy individuals who voluntarily provide a portion of their liver to a recipient. Donors have no medical indication for surgery and gain no procedural benefit from a hepatectomy. These patients voluntarily expose themselves to a lifethreatening procedure that is associated with a high rate of complications. ${ }^{3,4}$ Efforts to improve the safety and perioperative care of living liver donors are needed to ensure the viability of LLDT programsfuture donors may be dissuaded by the risk of morbidity and mortality. ${ }^{5}$

Postoperative pain is a significant source of morbidity after liver surgery. ${ }^{6}$ This is due to the large subcostal incisions that are required for surgical exposure. Approximately $63 \%$ of liver donors report more pain than anticipated after surgery. ${ }^{5}$ Although epidural analgesia is considered the gold standard to manage postoperative pain after major abdominal surgeries and has been found to be superior to intravenous (IV) patient-controlled analgesia (PCA), ${ }^{7-9}$ epidural use is associated with rare but significant adverse complications, such as epidural and spinal hematomas. Although no cases of spinal or epidural hematomas have been reported after LLDT, theoretically, there is an increased risk of this complication due to alterations in the coagulation profile after liver surgery. ${ }^{10,11}$ Thus, because of a theoretically increased risk, clinicians may be reluctant to place epidural catheters for living donor liver resection patients.

An alternative to epidural analgesia is increased IV opioid use. Opioids are effective for pain at rest; however, higher doses are required for movement-related pain. ${ }^{12}$ Furthermore, they are associated with side effects such as nausea, vomiting, sedation, and respiratory depression. ${ }^{13}$ Although IV lidocaine infusions have been shown to be effective for postoperative pain management in abdominal surgeries, their use in liver surgery is limited because of an increased potential for toxicity. ${ }^{14}$

An injection of local anesthetics within the transversus abdominis plane (TAP), known as the TAP block, has been shown to be an effective analgesic intervention in a variety of abdominal surgeries. ${ }^{15-17}$ A catheter placed along this plane and used for repeated bolus administration has been assessed in several small studies and case series. ${ }^{16,18-20}$ Previous studies used small sample sizes and often lacked a control group; this limited their ability to inform on the widespread use of this intervention.

Unlike TAP catheters placed with anatomical landmarks or ultrasound-guidance, LLDT catheters can be placed under direct vision at the end of the surgery so they can be located closer to the nociceptive pain fibers, and this may be associated with a lower rate of catheter failure. To avoid confusion, we refer to surgically placed TAP catheters as abdominal wall catheters (AWCs). These catheters are also identified as medial open transversus abdominal plane catheters in the literature. ${ }^{21}$ The primary aim of this study was to retrospectively compare the effects of AWCs after LLDT to the 2 standard postoperative pain modalities, IV PCA and patient-controlled epidural analgesia (PCEA), on postoperative pain scores and opioid consumption during the 3 postoperative days after surgery. Secondary aims of this study were to determine whether there are differences with respect to in-hospital recovery milestones and adverse events.

\section{PATIENTS AND METHODS}

This retrospective study was reviewed and approved by the research ethics board at the Toronto General Hospital (Toronto, Canada). Between April 2004 and December 2011, 340 consecutive patients who had undergone elective right lobe donor hepatectomy surgery were identified from institutional electronic databases. For patients undergoing orthotopic liver transplantation, AWCs were first used at our institution in April 2010 because of the surgeon's preference to reduce the hypotension associated with epidural analgesia after surgery.

\section{Preoperative Assessment}

All patients received a consultation with a liver transplant surgeon to determine eligibility and appropriateness for becoming a liver donor. Eligible patients subsequently underwent standardized assessments in the preoperative anesthesia consultation clinic. At this visit, information was provided regarding the postoperative pain intensity associated with an upper abdominal incision and the various pain modalities available, such as IV PCA, PCEA, and AWCs (if AWCs were available at the time). Known risks and benefits associated with each pain modality were discussed. All patients were informed that AWCs had not been formally studied but had a high safety profile in local quality assessment studies. We also disclosed potential risks such as catheter failure, infection, bleeding, and complications related to local anesthetic toxicity, such as blurry vision, dizziness, light-headedness, seizure, and cardiac failure.

\section{Intraoperative and Postoperative Management}

On the day of surgery, patients who consented to PCEA analgesia were brought into the operating room and placed in a sitting position on the operating room table. The standard site of epidural placement for this type of surgery ranged from thoracic spine level T7 to T10. After the placement of a flexible epidural catheter, placement was confirmed with a $3-\mathrm{mL}$ test dose of $2 \%$ lidocaine.

All patients were attached to standard Canadian Anesthesiology Society monitors before the induction 
of anesthesia. Anesthesia was induced with fentanyl, propofol, and a nondepolarizing muscle relaxant at doses deemed appropriate by the attending anesthesiologist. Upon the induction of anesthesia, an arterial cannula was placed in the radial artery for invasive blood pressure monitoring, and central venous access was obtained for central venous pressure monitoring. General anesthesia was maintained with a volatile anesthetic agent, and the goal was to keep the central venous pressure between 2 and $6 \mathrm{~mm} \mathrm{Hg}$. If a thoracic epidural had been placed, local anesthetic infusions were typically started intraoperatively. Epidurals were infused with a solution of $0.1 \%$ bupivacaine with $0.015 \mathrm{mg} / \mathrm{mL}$ of hydromorphone. Infusions were delivered with an Abbott GemStar infusion pump (Hospira, Lake Forest, IL) at a rate of $5 \mathrm{~mL} /$ hour. Patients were given intraoperative IV opioids at the discretion of the attending anesthesiologist.

Patients included in this study underwent elective right lobe donor hepatectomy surgery. All surgeries used a right subcostal oblique incision. The surgical technique was similar across different types of surgeons. Surgeons dissected out muscles of the anterior abdominal wall during surgical closure of the subcostal incision in patients receiving an AWC. They identified the plane between the internal oblique and transversus abdominis muscle, which is the TAP. A Tuohy needle was used to create 2 new insertions through the skin, and 2 multibore catheters (Portex epidural catheters, Smiths Medical, Markham, Canada) were placed along this plane deep into the fascial layer. One catheter was positioned medially in close proximity to the rectus sheath, and the other was placed in the right intercostal area. Catheters were placed to approximately cover the $\mathrm{T} 7$ to $\mathrm{T} 11$ intercostal nerve supply. The wound was then closed, with the 2 ends of the catheters secured in place with sterile dressings. A bolus of $0.125 \%$ bupivacaine 10.2 $\mathrm{mL} / \mathrm{kg}$ ) per catheter at the end of the operative case, before anesthesia emergence, was given at the discretion of the surgeon and the attending anesthesiologist.

Patients were extubated in the operating room postoperatively and were taken to the postanesthesia care unit. Patients in the IV PCA and AWC groups received a hand-held pendant connected to the IV PCA pump, whereas those in the PCEA group received a handheld pendant for an epidural infusion pump. There were standardized institutional protocols for those receiving IV PCA, PCEA, or AWCs postoperatively. The IV PCA protocol used either morphine $(1 \mathrm{mg} / \mathrm{mL})$ or hydromorphone $(0.4 \mathrm{mg} / \mathrm{mL})$. The IV PCA pumps were set to deliver a dose of 1 to $2 \mathrm{mg}$ of morphine with a 5-minute lockout period and a 4-hour maximum dose of $40 \mathrm{mg}$ or 0.2 to $0.4 \mathrm{mg}$ of hydromorphone with a 5-minute lockout period and a 4-hour maximum dose of $10 \mathrm{mg}$. Patients in the PCEA group received a $0.1 \%$ bupivacaine solution with $0.015 \mathrm{mg} / \mathrm{mL}$ of hydromorphone at an infusion rate of $5 \mathrm{~mL} /$ hour with a 3-mL bolus, a 20-minute lockout period, and a 4-hour maximum of $50 \mathrm{~mL}$. Patients in the AWC group received a bolus of $0.125 \%$ bupivacaine $(0.2 \mathrm{~mL} / \mathrm{kg})$ per catheter twice a day in addition to IV PCA as described previously. All patients were followed by the liver transplant team and the acute pain service (APS) postoperatively. The APS team visited each patient daily and modified the infusion/bolus protocol if pain control was not satisfactory or if adverse effects were significant. PCA, PCEA, and AWCs were removed when acute pain was minimal as determined by the APS team. Patients were then transferred to a breakthrough parental or oral opioid regimen. Most patients received oral acetaminophen every 6 hours when they were able to tolerate fluid sips.

Data were collected retrospectively after patients had been discharged from hospital. We used institutional databases of scanned electronic charts and archived APS records to retrieve data on each patient, including the following: postoperative pain modality; type and dose of intraoperative opioids, dexamethasone, and ketamine; intraoperative blood loss, fluid intake, and length of procedure; postoperative pain intensity as measured by a numeric rating scale (11point scale, with 0 indicating no pain and 10 indicating the worst possible pain) every 6 hours; daily IV opioid consumption in morphine equivalents (a conversion of 1:5 was used to convert hydromorphone to morphine) ${ }^{22}$; adverse effects (ie, nausea, vomiting, sedation, or pruritus); time to fluid sips and full diet; time to ambulation; and length of hospital stay. Outcomes were collected for the first 3 days after surgery.

Intraoperative opioids were separated into shortand long-acting opioids; long-acting opioids such as hydromorphone and morphine were combined to give a total intraoperative morphine-equivalent dose per patient, and fentanyl was recorded as a short-acting opioid. Sedation, nausea, vomiting, and pruritus were assessed by the attending step-down or intermediatecare nurse every 6 hours. Sedation was assessed with a 4-point scale: (0) alert, (1) mildly drowsy but easy to arouse, (2) moderately drowsy but easy to arouse, and (3) somnolent and difficult to arouse. If increased sedation became troublesome for patients in the PCEA group (sedation score of 3 for 2 days), the epidural solution was then changed to $0.2 \%$ ropivacaine without any opioids. If pruritus became troublesome for patients in any group, patients were treated with either $25 \mathrm{mg}$ of diphenhydramine or $25 \mathrm{mg}$ of hydroxyzine orally. If patients in the PCEA group required 2 or more doses of antipruritic medications, their epidural solutions were changed to $0.2 \%$ ropivacaine.

Demographic information, including sex, age, height, weight, surgeon, and date of surgery, were also obtained from the electronic medical records. The anesthetic record, APS notes, and progress notes were screened to determine whether a patient had a functioning or nonfunctioning epidural or AWCs. If a note described a malfunctioning (ie, leaking) or nonfunctioning epidural (ie, no pain relief with bolus medication) or AWCs that were not replaced, the patient was excluded from the study. If a malfunctioning or nonfunctioning catheter was removed and a new one was 
reinserted (ie, removing and replacing an epidural catheter or inserting new AWCs with anatomical landmarks/ultrasound guidance), the patient was classified according to the original group assignment. Crossovers (ie, patients started with IV PCA and subsequently switched to an epidural) were also excluded from this retrospective study. Furthermore, patients who received only 1 AWC were excluded.

\section{Statistical Analysis}

An analysis of postoperative outcomes was conducted on the basis of patients' postoperative pain modalities. The daily morphine-equivalent opioid consumption analysis was restricted to the IV PCA and AWC groups because those with well-functioning PCEAs invariably received substantially less opioids. A mixed-design analysis of variance (ANOVA) was used to assess the effect of pain modality on opioid use and the interaction between postoperative opioid consumption and time after surgery. Postoperative days $0,1,2$, and 3 were used as time points in this analysis. The data were tested for normality, and a log transformation was applied when necessary. Sedation scores were collapsed into a binary outcome where a score $>1$ indicated the presence of sedation and a score of 0 or 1 indicated its absence-this was to assess for an impact on clinically important sedation. A chi-square test was applied to determine differences between groups for categorical variables (sex, intraoperative ketamine or dexamethasone, nausea, vomiting, pruritus, and sedation), and a $t$ test for ANOVA was applied for the continuous variables [age, body mass index (BMI), intraoperative opioids, intraoperative fluids and blood loss, length of procedure, time to fluids and full diet, time to ambulation, and length of hospital stay]. The area under the pain curve (AUPC) for each group was calculated for 2 time periods: pain scores from postoperative day 0 at 1800 hours to postoperative day 1 at 2400 hours (time 1) and pain scores from postoperative day 1 at 2400 hours to postoperative day 3 at 0600 hours (time 2). Patients were excluded from the AUPC if they had 3 or more missing data points and if they had 2 consecutive missing pain level data points. The AUPC analysis was conducted with a nonparametric method.

A propensity score analysis was conducted to adjust for covariates and to confirm the primary analysis. The first step of the planned propensity score analysis was the estimation of the probability of receiving the treatment of interest (ie, IV PCA, PCEA, or AWC). An estimation of this probability was calculated with logistic regression modeling, which included the following baseline variables: age, sex, weight, height, intraoperative fentanyl, intraoperative morphine equivalence, intraoperative dexamethasone, intraoperative ketamine, surgeon, fluid intake, estimated blood loss, and length of the procedure. Propensity matching was performed with the nearest neighbor matching method with a caliper of $20 \%$ of the pooled standard deviation of the logit of the propensity score. Two propensity matches, matching patients in the AWC group to the IV PCA group and matching patients in the AWC and PCEA groups, were performed. No replacements in the matching were performed, and if patients in the non-AWC group were not matched to a patient in the AWC group, they were excluded from the matched populations.

Analyses were conducted with SPSS 20 (SPSS, Inc., Chicago, IL), and the propensity score match (PSM) and the analysis were performed with $\mathrm{R}$ Statistical Software 3.1.1 (R Foundation, Vienna, Austria). All tests used a level of significance of $\alpha=0.05$.

\section{RESULTS}

\section{Sample Size, Clinical Characteristics, and PSM}

A total of 340 patients were eligible for inclusion in this study. After the exclusion of nonfunctioning/malfunctioning epidurals or AWCs and crossovers, there were 167 patients in the IV PCA group, 68 patients in the PCEA group, and 84 patients in the AWC group. The exclusions included 21 patients in the AWC group who were excluded because of the placement of only 1 AWC, ${ }^{19}$ which was nonfunctioning and not replaced. ${ }^{2}$ Two epidurals were nonfunctioning but were later replaced, so those patients remained in the analysis.

Age, sex, BMI, intraoperative ketamine use, estimated blood loss, and length of procedure were similar across the 3 groups (Table 1). The PCEA group received fewer intraoperative morphine equivalents than the IV PCA and AWC groups. This was most likely due to intraoperative analgesia provided by epidural use. The IV PCA group received more intraoperative fentanyl, which may have been related to increased pain upon anesthetic emergence because those in the AWC group may have received a bolus of local anesthetics before extubation. Furthermore, more patients in the AWC group received intraoperative dexamethasone in comparison with the other groups. At our institution, dexamethasone is given as prophylaxis for postoperative nausea and vomiting (PONV), and more patients in the AWC group may have been at high risk for PONV and needed prophylaxis.

Two PSMs were performed, with adjustments for baseline covariates. Eighty-three patients in the AWC group were matched to 83 patients in the IV PCA group, whereas 26 patients in the AWC group were matched to 26 patients in the PCEA group.

\section{Opioid Consumption}

Figure 1 depicts the daily mean postoperative morphineequivalent opioid consumption for the IV PCA and AWC groups. Mean morphine-equivalent opioid consumption was significantly lower in the AWC group [18.1 mg, standard error $(\mathrm{SE})=3.1 \mathrm{mg}$ on day 3 versus the IV PCA group (28.2 mg, $\mathrm{SE}=3.0 \mathrm{mg}$ ). Mean cumulative morphine-equivalent opioid consumption was also lower in the AWC group (97.2 $\mathrm{mg}, \mathrm{SE}=7.2 \mathrm{mg}$ ) versus the IV PCA group (121.0 mg, $\mathrm{SE}=9.1 \mathrm{mg}$ ). The mixed-design ANOVA with log-adjusted opioid consumption data indicated an interaction between postoperative time and morphine-equivalent opioid consumption for patients in the AWC group. PSMs demonstrated similar differences 


\begin{tabular}{|c|c|c|c|}
\hline Variable & Group 1: IV PCA & Group 2: PCEA & Group 3: AWC \\
\hline Age (years) & $36.2 \pm 11.4$ & $36.2 \pm 11.1$ & $35.6 \pm 12.0$ \\
\hline Sex (male/female) & $81 / 86$ & $28 / 40$ & $40 / 44$ \\
\hline BMI $\left(\mathrm{kg} / \mathrm{m}^{2}\right)$ & $25.2 \pm 3.9$ & $25.4 \pm 3.8$ & $26.2 \pm 4.1$ \\
\hline $\begin{array}{l}\text { Intraoperative morphine equivalents } \\
\text { [mg; mean (SE)] }\end{array}$ & $9.8(0.6)$ & $2.1(0.4)^{*}$ & $8.6(0.7)$ \\
\hline Intraoperative fentanyl [ $\mu g ;$ mean (SE)] & $473.2(16.0)^{*}$ & $311.3(20.7)$ & $350.0(15.5)$ \\
\hline Intraoperative dexamethasone [n (\%)] & $23(13.8)$ & $3(4.4)$ & $29(34.5)^{\dagger}$ \\
\hline Intraoperative ketamine [n (\%)] & $0(0)$ & $1(1.5)$ & $7(8.3 \%)$ \\
\hline Intraoperative fluid intake [mL; mean (SE)] & $4592.2(134.5)$ & $4710.4(202.4)$ & $3360(122.8)^{\dagger}$ \\
\hline Estimated blood loss [mL; mean (SE)] & $895.0(52.9)$ & $950.9(83.0)$ & $752.4(103.3)$ \\
\hline Length of procedure [minutes; mean (SE)] & $364.1(5.7)$ & $361.5(8.4)$ & $350.1(7.6)$ \\
\hline Surgeon $(\mathrm{A} / \mathrm{B} / \mathrm{C} / \mathrm{D} / \mathrm{E} / \mathrm{F} / \mathrm{G})$ & $50 / 0 / 43 / 32 / 39 / 1 / 2$ & $17 / 0 / 19 / 18 / 14 / 0 / 0$ & $26 / 2 / 36 / 0 / 6 / 9 / 5$ \\
\hline
\end{tabular}

in morphine-equivalent opioid consumption in the unmatched data for all days; however, there was a loss of significance in the difference in the postoperative day 3 consumption (AWC, $16.6 \mathrm{mg}, \mathrm{SE}=2.7 \mathrm{mg}$ versus IV $\mathrm{PCA}, 23.8 \mathrm{mg}, \mathrm{SE}=3.7 \mathrm{mg} ; P=0.135)$. The difference in cumulative morphine-equivalent opioid consumption remained significant in the propensity-matched data (AWC, $98.0 \mathrm{mg}, \mathrm{SE}=7.4 \mathrm{mg}$ versus IV PCA, $136.1 \mathrm{mg}$, $\mathrm{SE}=17.0 \mathrm{mg} ; P=0.04$ ).

\section{Pain Scores}

Figure 2 depicts the pain scores for each group during the first 3 postoperative days. The AUPC analysis included 197 patients (88 IV PCA patients, 46 PCEA patients, and 63 AWC patients); 122 patients were excluded because of a priori conditions. The AUPC was not significantly different in patients who received AWCs versus IV PCA throughout both time $1(P=$ 0.332) and time $2(P=0.059)$. The AUPC was significantly smaller for patients who received PCEA versus AWC for both time $1(P<0.001)$ and time $2(P=$ 0.006). These findings were similar when the analysis was conducted with the PSM data.

\section{Oral Intake}

The time to begin tolerating fluid sips postoperatively was significantly longer in the AWC group (2.0 days, $\mathrm{SE}=0.11$ days) versus the IV PCA group (1.6 days, $\mathrm{SE}=0.09$ days; $P=0.02$ ) but was similar to the time for the PCEA group (1.6 days, $\mathrm{SE}=0.14$ days; Table 2). In contrast, the AWC group advanced to a full diet earlier than either the IV PCA group (3.5 versus 3.9 days;

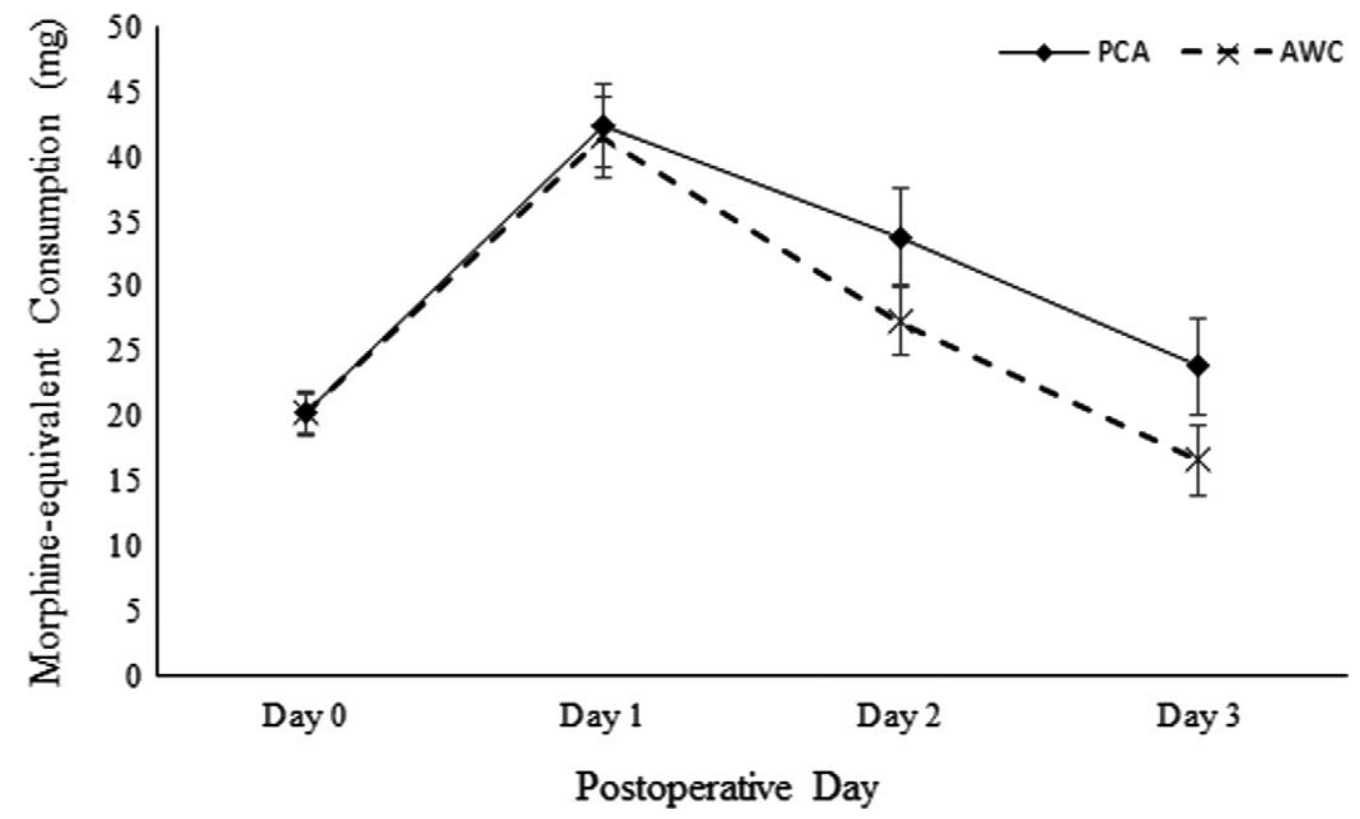

Figure 1. Postoperative morphine-equivalent opioid consumption in the first 3 days after surgery in the IV PCA and AWC groups. 


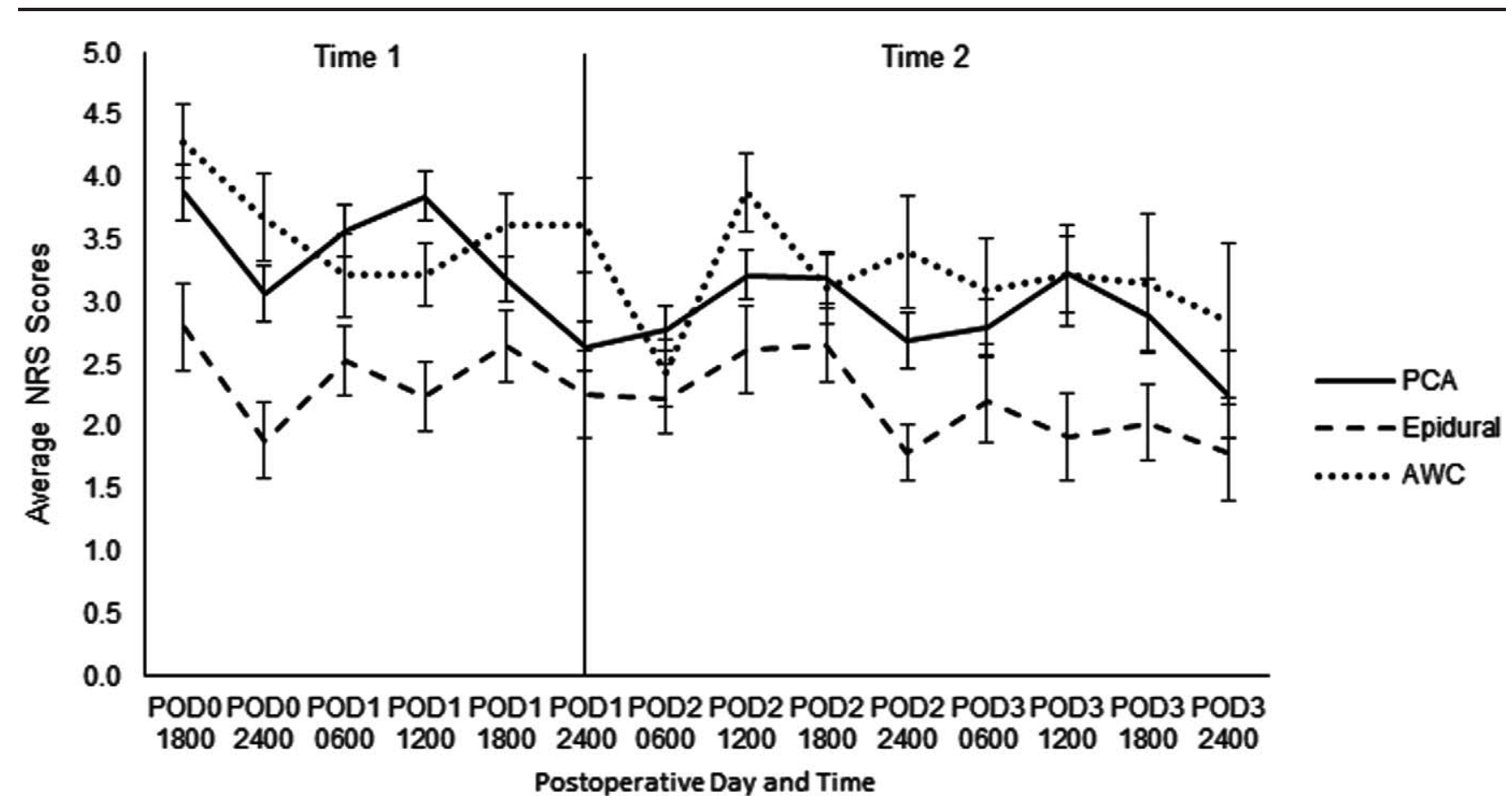

Figure 2. Postoperative pain scores in the first 3 days after surgery in the IV PCA, PCEA, and AWC groups.

$P=0.045$ ) or the PCEA group (4.2 days; $P=0.003$ )

Findings were similar with the PSM data.

\section{Time to Ambulation}

There was no difference in the time to ambulation between the 3 groups in the unmatched data (Table 2) and the propensity-matched data.

\section{Length of Hospital Stay}

There was no difference in the length of hospital stay between the AWC group and the IV PCA group $(P=$ 0.071 ; Table 2). The length of hospital stay was significantly less for the AWC group (6.0 days, $\mathrm{SE}=0.12$ days) versus the PCEA group (6.6 days, $\mathrm{SE}=0.22$ days; $P=0.028$ ); however, this finding was nonsignificant with the PSM data $(P=0.32)$.

\section{Adverse Events}

Eight patients (7.5\%) in the AWC group were documented to have clinically important sedation (sedation score $\geq 2$ ) at some point during the first 3 postopera- tive days. This was a significantly lower proportion than that found in the IV PCA group $(70.8 \%$; $P<$ $0.001)$ and the PCEA group $(21.7 \% ; P<0.001)$. Furthermore, those in the PCEA group had significantly more pruritus than those in the AWC group $(P=0.004)$ and those in the IV PCA group $(P=$ $0.001)$. There was no difference in the incidence of PONV between the 3 groups (Table 3). The findings were similar with the PSM data.

\section{DISCUSSION}

Pain is a significant concern for patients undergoing surgery. ${ }^{23}$ Severe postoperative pain negatively influences a patient's satisfaction with his or her surgery and anesthetic care. ${ }^{24}$ Furthermore, living liver donor safety and satisfaction are important in order to ensure the viability and longevity of adult donor programs. During the past 10 years, there has been a reduction in the number of living liver donors. ${ }^{25}$ Postoperative pain is a disincentive and potentially a factor contributing to this decline. It is important that efforts are invested to improve care and reduce

TABLE 2. Postoperative Recovery Milestones and Length of Hospital Stay for the IV PCA, PCEA, and AWC Groups

\begin{tabular}{lrrr}
\hline Variable & $\begin{array}{r}\text { Group 1: IV PCA } \\
\text { [Mean (SE)] }\end{array}$ & $\begin{array}{r}\text { Group 2: PCEA } \\
\text { [Mean (SE)] }\end{array}$ & $\begin{array}{r}\text { Group 3: AWC } \\
\text { [Mean (SE)] }\end{array}$ \\
\hline Time to fluid sips (days) & $1.6(0.09)^{*}$ & $1.6(0.14)$ & $2.0(0.11)$ \\
Time to full diet (days) & $3.9(0.09)^{*}$ & $4.2(0.21)^{*}$ & $3.5(0.12)$ \\
Time to ambulation (days) & $1.8(0.09)$ & $2.0(0.15)$ & $1.7(0.13)$ \\
Length of hospital stay (days) & $6.4(0.13)$ & $6.6(0.22)^{*}$ & $6.0(0.12)$ \\
\hline
\end{tabular}

$* P<0.001$ versus the AWC group. 


\begin{tabular}{|c|c|c|c|}
\hline \multicolumn{4}{|c|}{$\begin{array}{l}\text { TABLE 3. Postoperative Adverse Events Within the } \\
\text { First } 3 \text { Days After Surgery in the IV PCA, PCEA, and } \\
\text { AWC Groups }\end{array}$} \\
\hline Variable & $\begin{array}{l}\text { Group 1: IV } \\
\text { PCA [n (\%)] }\end{array}$ & $\begin{array}{r}\text { Group 2: } \\
\text { PCEA [n (\%)] }\end{array}$ & $\begin{array}{r}\text { Group 3: } \\
\text { AWC [n (\%)] }\end{array}$ \\
\hline Nausea & $43(25.7)$ & $21(30.9)$ & $27(32.1)$ \\
\hline Vomiting & $9(5.4)$ & $5(7.4)$ & $6(7.1)$ \\
\hline Pruritus & $28(16.8)$ & $26(38.2)^{*}$ & 14 (16.7) \\
\hline Sedation & $75(44.9)^{\dagger}$ & $23(33.8)^{\dagger}$ & 8 (9.5) \\
\hline
\end{tabular}

complications for those generous enough to donate their organs.

There are 2 main strengths to our study. First, it provides the largest investigation of catheters placed along the TAP to date. Second, we have compared a novel pain intervention to 2 standard-of-care modalities: IV PCA and PCEA.

Findings from our study indicate that AWCs in living liver donors provide an opioid-sparing effect in comparison with patients using IV PCA alone after postoperative day 2. There also appears to be an interaction between opioid reduction and the time after surgery. This interaction effect may be partially explained by synergism between the natural history of postoperative pain (ie, postoperative pain improves with time) and the pain-reducing effect of AWCs. Pain scores for patients receiving AWCs were similar to those for patients receiving IV PCA and higher than those for patients receiving epidural analgesia. At our institution, we encourage patients to use their IV PCA to keep their pain scores below $4 / 10$ or within a level of acceptable pain relief. Because patients in the AWC group also received IV PCA, it is not surprising that these 2 groups had similar pain scores. Although the literature already indicates that epidural analgesia is superior to IV PCA, our results suggest that epidural analgesia is also superior to AWCs with back-up IV PCA.

Despite epidural analgesia being considered the gold standard for postoperative pain management after open abdominal surgeries, it is used with caution in patients undergoing liver surgery. ${ }^{8,14}$ Patients suffering from liver failure have significant reductions in platelet numbers and changes in their coagulation profile. ${ }^{10,11}$ Although living liver donors are relatively healthy, the potential risk for catastrophic permanent neurological complications to occur after a spinal or epidural hematoma may have dissuaded the use of epidural use in these patients. Moreover, local anesthetics and/or opioids delivered through epidural infusions are associated with other adverse complications such as sedation, pruritus, urinary retention, and arterial hypotension. $8,26,27$ Because of the riskto-benefit profile associated with epidural catheter placement, AWCs may provide an option for patients who are concerned about complications associated with epidural analgesia or for whom epidurals are contraindicated.

AWCs provide their analgesic effects by infusing local anesthetics into the same anatomical space where the nerves responsible for incisional postoperative pain reside. ${ }^{28}$ Injecting or infusing local anesthetics into the TAP has gained popularity in recent years. Initially described as a landmark technique to be used after lower abdominal surgeries, ${ }^{29}$ variations of the TAP block have been used in various open, closed, upper, and lower abdominal surgeries. Previous systematic reviews and meta-analyses on perioperative TAP blocks suggest effective postoperative pain control. ${ }^{15,30}$ However, only a few studies have explored the use of TAP blocks or AWCs in liver resection patients. Niraj et al. ${ }^{31}$ conducted a randomized controlled trial in liver and kidney surgical patients comparing TAP catheters to epidural analgesia. They found that TAP catheters resulted in equivalent pain scores on coughing at 8 and 72 hours postoperatively. However, this study lacked an analysis of side effects and hospital-based outcomes. Although the results from a recent study by Behman et al. $^{21}$ suggest that surgically placed catheters in liver resection patients can provide effective pain relief after surgery, the study did not include a control group, and this precludes a true estimate of their efficacy. Nonetheless, these studies, in addition to our investigation, provide an impetus for future prospective trials in this area.

Our study demonstrates that the opioid-sparing effect of AWCs may result in a reduction in opioidrelated side effects. AWCs were associated with less sedation than epidural analgesia and IV PCA alone. The sedative effects of opioids are more pronounced in those after liver resection because of transient impairment of liver function and altered opioid metabolism; thus, any reduction in opioid consumption may be clinically significant. ${ }^{32}$ It is also important to minimize the sedative properties of narcotics after surgery because these symptoms can be similar to those of hepatic insufficiency, which may require urgent surgical intervention. ${ }^{6}$ Epidural analgesia was also associated with increased pruritus in this study. Increased pruritus is a common side effect of epidural analgesia, and it is believed to be due to the use of epidural opioids. ${ }^{33-35}$ It should be noted that patients in this study received hydromorphone in their epidural infusions, which is known to cause pruritus and sedation. Thus, an increase in pruritus and sedation in the PCEA group in the study may be due to our institution's use of epidural opioids. By avoiding opioids in the epidural space and reducing parenteral opioid consumption, AWCs provide an alternative for those concerned about postoperative side effects.

There is emerging evidence that epidural analgesia is associated with a decrease in postoperative ileus. $^{8,36}$ The biological mechanism of action is believed to be due to a stimulant effect of systemically absorbed local anesthetics on the gastrointestinal 
system. ${ }^{37}$ Because AWCs also use local anesthetics, we examined their effect on surrogates of gastrointestinal function (time to fluid and oral intake). Although AWCs were associated with an increased time to fluid sips, they were ultimately associated with a reduction in the time to tolerate a full diet. To that extent that systemic local anesthetic absorption in the AWC group expedited gastrointestinal function, it is unclear why advancement of diet was not shorter in the PCEA group versus the IV PCA group (3.9 days with IV PCA versus 4.2 days with PCEA). It is possible that the prokinetic effect of systemically absorbed local anesthetics in the PCEA group may have been negated by systemically absorbed opioids from the epidural solution. $^{38}$ Nonetheless, reduced opioid-related side effects and a decreased time to tolerate a full diet in the AWC group possibly contributed to a reduced length of hospital stay in comparison with the PCEA group in the unmatched data. Reducing the time to normal bowel function and the length of hospital stay could have tremendous implications for hospital and health care expenditures if these trends prove to be consistent in large randomized controlled trials.

We believe that the surgical placement of AWCs has several advantages. First, it enables active surgical participation in the patient's postoperative pain management. Second, it may allow improved placement of catheters due to direct visualization. In the study of Niraj et al., ${ }^{31}$ a $63 \%$ success rate was achieved when TAP catheters were placed under ultrasound guidance. Our study obtained a 97\% success rate, which was similar to the study results of Behman et al., ${ }^{21}$ who achieved a $94 \%$ success rate with the surgical placement of catheters. Third, it prevents avoid complications such as liver puncture or hematoma formation associated with catheter placement via anatomical landmarks or ultrasound guidance. ${ }^{39}$

Although our study suggests several benefits of surgically placed AWCs after living liver donation, there are several limitations to our investigation. First, this study was a retrospective cohort study and was vulnerable to several methodological issues, such as recall bias and missing data. Second, this study included patients from a 6-year period. Although significant changes in perioperative management during this time period did not occur, slight changes could have contributed to additional or immeasurable confounding effects in this study. Third, because this study sought to determine the efficacy of AWCs, those with nonworking or partially working catheters were excluded. Although this may have introduced a selection bias, we believe that this allowed a better assessment of the efficacy of the analgesic technique. Finally, the propensity analysis excluded patients who did not find a match in the treatment group, and this reduced the statistical power. Thus, differences such as postoperative day 3 opioid consumption and length of stay in the unmatched data may have been significantly different, but there was reduced statistical power to detect an effect in the propensity analysis.
Findings from this study suggest several potential benefits of AWCs, including a reduction in postoperative opioid consumption, reduced adverse events, an improved time to tolerate a full diet, and possibly a decreased length of hospital stay. Although epidural analgesia continues to provide the gold standard of pain relief after surgery, AWCs appear to be a safe adjunct to IV PCA after living liver donation. Future prospective investigations on AWCs are needed before their widespread and routine use can be advocated. Large randomized controlled trials are needed to verify potential benefits and determine safety and adverse events associated with AWC use.

\section{REFERENCES}

1. Florman S, Miller CM. Live donor liver transplantation. Liver Transpl 2006; 12:499-510.

2. Bachir NM, Larson AM. Adult liver transplantation in the United States. Am J Med Sci 2012; 343:462-469.

3. Beavers KL, Sandler RS, Shrestha R. Donor morbidity associated with right lobectomy for living donor liver transplantation to adult recipients: a systematic review. Liver Transpl 2002; 8:110-117.

4. Jeon H, Lee SG. Living donor liver transplantation. Curr Opin Organ Transplant 2010; 15:283-287.

5. Verbesey JE, Simpson MA, Pomposelli JJ, Richman E, Bracken AM, Garrigan K, et al. Living donor adult liver transplantation: a longitudinal study of the donor's quality of life. Am J Transplant 2005; 5:2770-2777.

6. Hwang GS, McCluskey SA. Anesthesia and outcome after partial hepatectomy for adult-to-adult donor transplantation. Curr Opin Organ Transplant 2010; 15:377-382.

7. Kehlet H. Postoperative ileus-an update on preventive techniques. Nat Clin Pract Gastroenterol Hepatol 2008; 5:552-558.

8. Marret E, Remy C, Bonnet F; for Postoperative Pain Forum Group. Meta-analysis of epidural analgesia versus parenteral opioid analgesia after colorectal surgery. $\mathrm{Br} \mathrm{J}$ Surg 2007; 94:665-673.

9. Clarke H, Chandy T, Srinivas C, Ladak S, Okubo N, Mitsakakis $\mathrm{N}$, et al. Epidural analgesia provides better pain management after live liver donation: a retrospective study. Liver Transpl 2011;17:315-323.

10. Borromeo CJ, Stix MS, Lally A, Pomfret EA. Epidural catheter and increased prothrombin time after right lobe hepatectomy for living donor transplantation. Anesth Analg 2000; 91:1139-1141.

11. Choi SJ, Gwak MS, Ko JS, Kim GS, Ahn HJ, Yang M, et al. The changes in coagulation profile and epidural catheter safety for living liver donors: a report on 6 years of our experience. Liver Transpl 2007; 13:62-70.

12. Nimmo SM. Benefit and outcome after epidural analgesia. Contin Educ Anaesth Crit Care Pain 2004; 4:44-47.

13. Mann C, Pouzeratte Y, Boccara G, Peccoux C, Vergne C, Brunat $\mathrm{G}$, et al. Comparison of intravenous or epidural patient-controlled analgesia in the elderly after major abdominal surgery. Anesthesiology 2000; 92:433-441.

14. Thomson PD, Melmon KL, Richardson JA, Cohn K, Steinbrunn W, Cudihee R, Rowland M. Lidocaine pharmacokinetics in advanced heart failure, liver disease, and renal failure in humans. Ann Intern Med 1973; 78:499-508.

15. Abdallah FW, Chan VW, Brull R. Transversus abdominis plane block: a systematic review. Reg Anesth Pain Med 2012; 37:193-209.

16. Bjerregaard N, Nikolajsen L, Bendtsen TF, Rasmussen BS. Transversus abdominis plane catheter bolus 
analgesia after major abdominal surgery. Anesthesiol Res Pract 2012; 2012:596536.

17. Wahba SS, Kamal SM. Analgesic efficacy and outcome of transversus-abdominis plane block versus low thoracicepidural analgesia after laparotomy in ischemic heart disease patients. J Anesth 2014; 28:517-523.

18. Basu S, Tamijmarane A, Bulters D, Wells JK, John TG, Rees M. An alternative method of wound pain control following hepatic resection: a preliminary study. HPB (Oxford) 2004; 6:186-189.

19. Taylor LJ, Birmingham P, Yerkes E, Suresh S. Children with spinal dysraphism: transversus abdominis plane (TAP) catheters to the rescue! Paediatr Anaesth 2010; 20: 951-954.

20. Niraj G, Kelkar A, Fox AJ. Oblique sub-costal transversus abdominis plane (TAP) catheters: an alternative to epidural analgesia after upper abdominal surgery. Anaesthesia 2009; 64:1137-1140.

21. Behman R, McHardy P, Sawyer J, Lam-McCulloch J, Karanicolas PJ. Medial open transversus abdominal plane catheter analgesia: a simple, safe, effective technique after open liver resection. J Am Coll Surg 2014; 218:e91-94.

22. Patanwala AE, Duby J, Waters D, Erstad BL. Opioid conversions in acute care. Ann Pharmacother 2007;41:255266.

23. Apfelbaum JL, Chen C, Mehta SS, Gan TJ. Postoperative pain experience: results from a national survey suggest postoperative pain continues to be undermanaged. Anesth Analg 2003;97:534-540.

24. Myles PS, Williams DL, Hendrata M, Anderson H, Weeks AM. Patient satisfaction after anaesthesia and surgery: results of a prospective survey of 10,811 patients. Br J Anaesth 2000;84:6-10.

25. Organ Procurement and Transplantation Network (OPTN) and Scientific Registry of Transplant Recipients (SRTR). OPTN/SRTR 2011 Annual Data Report. Rockville, MD: Department of Health and Human Services, Health Resources and Services Administration, Healthcare Systems Bureau, Division of Transplantation; 2012.

26. Giebler RM, Scherer R, Peters J. Incidence of neurologic complications related to thoracic epidural catheterization. Anesthesiology 1997;86:55-63.

27. Biebuyck J. Epidural anesthesia and analgesia: Their role in postoperative outcome. Anesthesiology 1995;82: 1474-1506.
28. Rozen WM, Tran TMN, Ashton MW, Barrington MJ, Ivanusic JJ, Taylor GI. Refining the course of the thoracolumbar nerves: a new understanding of the innervation of the anterior abdominal wall. Clin Anat 2008; 21:325-333.

29. McDonnell JG, O’Donnell B, Curley G, Heffernan A, Power C, Laffey JG. The analgesic efficacy of transversus abdominis plane block after abdominal surgery: a prospective randomized controlled trial. Anesth Analg 2007; 104:193-197.

30. Charlton S, Cyna AM, Middleton P, Griffiths JD. Perioperative transversus abdominis plane (TAP) blocks for analgesia after abdominal surgery. Cochrane Database Syst Rev 2010;12:CD007705.

31. Niraj G, Kelkar A, Jeyapalan I, Graff-Baker P, Williams $\mathrm{O}$, Darbar A, et al. Comparison of analgesic efficacy of subcostal transversus abdominis plane blocks with epidural analgesia following upper abdominal surgery. Anaesthesia 2011;66:465-471.

32. Gholson C, Provenza J, Bacon B. Hepatologic considerations in patients with parenchymal liver disease undergoing surgery. Am J Gastroenterol 1990;85:487-496.

33. Szarvas S, Harmon D, Murphy D. Neuraxial opioid-induced pruritus: a review. J Clin Anesth 2003;15:234-239.

34. Hodgson PS, Liu SS, Gras TW. Does epidural anesthesia have general anesthetic effects? A prospective, randomized, double-blind, placebo-controlled trial. Anesthesiology 1999;91:1687-1692.

35. Chaney MA. Side effects of intrathecal and epidural opioids. Can J Anaesth 1995;42:891-903.

36. Vigneault L, Turgeon AF, Côté D, et al. Perioperative intravenous lidocaine infusion for postoperative pain control: a meta-analysis of randomized controlled trials. Can J Anaesth 2011;58:22-37.

37. Ness TJ. Intravenous lidocaine inhibits visceral nociceptive reflexes and spinal neurons in the rat. Anesthesiology 2000;92:1685-1691.

38. Jorgensen H, Fomsgaard JS, Dirks J, Wetterslev J, Andreasson B, Dahl JB. Effect of epidural bupivacaine vs combined epidural bupivacaine and morphine on gastrointestinal function and pain after major gynaecological surgery. Br J Anaesth 2001;87:727-732.

39. Milan ZB, Duncan B, Rewari V, Kocarev M, Collin R. Subcostal transversus abdominis plane block for postoperative analgesia in liver transplant recipients. Transplant Proc 2011; 43:2687-2690. 